\title{
Article \\ On the Complex-Valued Distribution Function of Charged Particles in Magnetic Fields
}

\author{
Andrey Saveliev 1,2 (D)
}

check for

updates

Citation: Saveliev, A. On the Complex-Valued Distribution Function of Charged Particles in Magnetic Fields. Mathematics 2021, 9, 2382. https://doi.org/10.3390/ math9192382

Academic Editor: Manuel Manas

Received: 2 September 2021

Accepted: 23 September 2021

Published: 25 September 2021

Publisher's Note: MDPI stays neutral with regard to jurisdictional claims in published maps and institutional affiliations.

Copyright: (C) 2021 by the author. Licensee MDPI, Basel, Switzerland. This article is an open access article distributed under the terms and conditions of the Creative Commons Attribution (CC BY) license (https:// creativecommons.org/licenses/by/ $4.0 /)$.
1 Institute of Physics, Mathematics and Information Technology, Immanuel Kant Baltic Federal University, 236016 Kaliningrad, Russia; andrey.saveliev@desy.de

2 Faculty of Computational Mathematics and Cybernetics, Lomonosov Moscow State University, 119991 Moscow, Russia

\begin{abstract}
In this work, we revisit Boltzmann's distribution function, which, together with the Boltzmann equation, forms the basis for the kinetic theory of gases and solutions to problems in hydrodynamics. We show that magnetic fields may be included as an intrinsic constituent of the distribution function by theoretically motivating, deriving and analyzing its complex-valued version in its most general form. We then validate these considerations by using it to derive the equations of ideal magnetohydrodynamics, thus showing that our method, based on Boltzmann's formalism, is suitable to describe the dynamics of charged particles in magnetic fields.
\end{abstract}

Keywords: Boltzmann equation; distribution function; fluid dynamics; kinetic consistent schemes; magnetic fields; magnetohydrodynamics

1 Introduction 1

2 The Distribution Function and the Boltzmann Equation 2

3 The Complex-Valued Distribution Function 4

3.1 Motivation . . . . . . . . . . . . . . . . . . . 4

3.2 The Complex-Valued Maxwellian Distribution Function . . . . . . . . . . 5

3.3 Moments of the Complex-Valued Boltzmann Distribution Function . . . . . . 6

3.4 Moments of the Boltzmann Equation for the Complex-Valued Distribution Function ...................... 8

4. Conclusions and Outlook $\quad 10$

References

\section{Introduction}

The main quantity considered in the kinetic theory of gases is the distribution function for which the time evolution is governed by the Boltzmann equation. Both together are used to describe the microscopic properties of gases by extracting the relevant quantities and their dynamics by calculating the corresponding moments [1].

Many important problems in modern fields of physics require not only the study of the dynamics of gases consisting of neutral particles, but also the consideration of fluids consisting of charged particles in electric and magnetic fields, such as electron clouds, ionized gas, plasma, ionized interstellar matter in astrophysics and others.

Up to now, the main approach to study the kinetics of charged particles is the direct combination of kinetic and electromagnetic equations. Initially, this was suggested by Vlasov, who included electromagnetic terms in the kinetic equation, thus deriving the Vlasov equations [2]. In this approach, electromagnetic forces are introduced phenomenologically as external Lorentz forces and require the solution of the full set of Maxwell 
equations. However, there have been also attempts to implement the electromagnetic forces directly into the distribution function, even though this kind of approach always has to consider the difficulty that magnetic field forces behave like axial vectors. In [3], a flux-splitting approach was suggested, focusing, however, more on the computational aspects rather than the physical background. Further progress was made by [4] who tried to incorporate the magnetic field into the distribution function. This, however, also required a modification of the transport equation such that the magnetic field cannot be regarded as an intrinsic part of the kinetic formalism, as is the case in the work presented here. Finally, in [5], based on [6], it was suggested to introduce a separate distribution function for the magnetic field and then to analyze it, using specific lattice kinetic schemes.

In previous works of the author of the present paper and his collaborators, the basic idea of a complex-valued distribution function [7] was introduced. Then, in [8-10], we derived and analyzed the kinetically consistent scheme to solve the resulting time evolution equations, while in later works, we focused on the numerical implementation, specifically on high-performance computational systems [11,12].

In this paper, we present the theoretical foundation and the general form of the extended complex-valued distribution function. On the one hand, this includes the derivation of the physical terms from first principles of the kinetic theory. On the other hand, we prove the validity of this distribution function through the calculation of the correct magnetohydrodynamic (MHD) quantities and the corresponding equations via its moments (the magnetic field now being one of them) and the moments of the Boltzmann equation, respectively.

Another reason for this study is that, despite the known difficulties faced by any attempt to solve the Boltzmann equation, nowadays, it is possible to explore its solution, using numerical methods on high-performance computational systems. The two most promising approaches are, on the one hand, the lattice Boltzmann schemes (LBS), which use numerical schemes for the study of the evolution of the distribution function and calculate the macroscopic quantities as its moments [13], and, on the other hand, the kinetic consistent schemes (KCS), which derive the hydrodynamic equations from the Boltzmann equation [14]. Therefore, any further progress in this field is based on the development of algorithms for the numerical solution of problems in physics, using LBS and KCS [8].

This paper is organized as follows: In Section 2, we briefly recapitulate the general concepts of the distribution function and the corresponding Boltzmann equation. Subsequently, in Section 3, we introduce and analyze the novel, complex-valued distribution function in order to directly include magnetic fields in the most natural and intuitive way. Finally, in Section 4, we summarize our findings and provide a short outlook on possible future developments.

\section{The Distribution Function and the Boltzmann Equation}

In this section, we want to recapitulate the basic concepts of the distribution function and the Boltzmann equation. The basis of these concepts was first introduced by Boltzmann in 1872 [15], and the following discussion may be found in various textbooks on kinetic theory, for example $[14,16]$

The kinetic theory describes the dynamics of gases via the time evolution of the distribution function $f(\mathbf{x}, \xi, t)$ (where $\mathbf{x}$ and $\mathcal{\xi}$ denote elements of the single-particle position and momentum phase space, respectively), which is governed by the Boltzmann equation [1]:

$$
\frac{\partial f(\mathbf{x}, \boldsymbol{\xi}, t)}{\partial t}+\boldsymbol{\xi} \cdot \nabla f(\mathbf{x}, \boldsymbol{\xi}, t)=C(f),
$$

where $C(f)$ is the collision integral, a non-linear integral operator describing the collisions of particles. Note that we consider the case without external forces, as our goal is to show that the formalism presented in this work naturally includes magnetic fields as an internal contribution to the distribution function. 
The equilibrium state of gases is described by a Maxwellian distribution function for which the collision integral vanishes. In order to obtain this distribution function at equilibrium, we use the Boltzmann $H$-theorem $[15,17]$ for which the relevant quantities are discussed in the following. Assuming the charged particles to be spherical, the gas state is uniform and the velocity distribution function $f$ is independent of $x, y, z$. In this case, the Boltzmann equation reduces to the following:

$$
\frac{\partial f}{\partial t}=\int\left(f^{\mathrm{fin}} f^{1, \text { fin }}-f f^{1}\right) v_{\text {rel }} \mathrm{d} \sigma \mathrm{d}^{3} v^{1},
$$

where $v_{\text {rel }}=\left|\mathbf{v}-\mathbf{v}^{1}\right|$ is the relative initial velocity between the two colliding particles, $\mathrm{d} \sigma$ is the differential collision cross section, $\mathbf{v}^{1}$ is the velocity of the second particle, and the superscript ${ }^{\text {fin }}$ symbol indicates the final states after the collision.

According to the Boltzmann $H$-theorem, the distribution function should, at equilibrium, minimize the integral quantity $H$ given by the following [15]:

$$
H=\int f \ln f \mathrm{~d}^{3} v
$$

from which we obtain the following:

$$
\frac{\mathrm{d} H}{\mathrm{~d} t}=\int \frac{\partial}{\partial t}(f \ln f) \mathrm{d}^{3} v=\int(1+\ln f) \frac{\partial f}{\partial t} \mathrm{~d}^{3} v .
$$

Plugging in Equation (2) provides the following:

$$
\begin{aligned}
\frac{\mathrm{d} H}{\mathrm{~d} t} & =\int(1+\ln f)\left[\int\left(f^{\mathrm{fin}} f^{1, \text { fin }}-f f^{1}\right) v_{\text {rel }} \mathrm{d} \sigma \mathrm{d}^{3} v^{1}\right] \mathrm{d}^{3} v \\
& =\frac{1}{2} \int\left(2+\ln f+\ln f^{1}\right)\left[\int\left(f^{\mathrm{fin}} f^{1, \text { fin }}-f f^{1}\right) v_{\text {rel }} \mathrm{d} \sigma \mathrm{d}^{3} v^{1}\right] \mathrm{d}^{3} v \\
& =\frac{1}{2} \int\left[2+\ln \left(f f^{1}\right)\right]\left(f^{\text {fin }} f^{1, \text { fin }}-f f^{1}\right) v_{\text {rel }} \mathrm{d} \sigma \mathrm{d}^{3} v^{1} \mathrm{~d}^{3} v \\
& =\frac{1}{4} \int\left[2+\ln \left(f f^{1}\right)\right]\left(f^{\mathrm{fin}} f^{1, \text { fin }}-f f^{1}\right) \\
& +\left[2+\ln \left(f^{\mathrm{fin}} f^{1, \text { fin }}\right)\right]\left(f f^{1}-f^{\mathrm{fin}} f^{1, \text { fin }}\right) v_{\text {rel }} \mathrm{d} \sigma \mathrm{d}^{3} v^{1} \mathrm{~d}^{3} v \\
& =\frac{1}{4} \int\left[\ln \left(f f^{1}\right)-\ln \left(f^{\mathrm{fin}} f^{1, \text { fin }}\right)\right]\left(f^{\mathrm{fin}} f^{1, \text { fin }}-f f^{1}\right) v_{\text {rel }} \mathrm{d} \sigma \mathrm{d}^{3} v^{1} \mathrm{~d}^{3} v \\
& =\frac{1}{4} \int \ln \left(\frac{f f^{1}}{f^{\mathrm{fin}} f^{1, \text { fin }}}\right)\left(f f^{1}-f^{\text {fin }} f^{1, \text { fin }}\right) v_{\text {rel }} \mathrm{d} \sigma \mathrm{d}^{3} v^{1} \mathrm{~d}^{3} v
\end{aligned}
$$

At equilibrium without external forces, according to the $H$-theorem, we have the following:

$$
\frac{\mathrm{d} H}{\mathrm{~d} t}=0,
$$

from which the following relation is obtained.

$$
\ln f_{0}+\ln f_{0}^{1}=\ln f_{0}^{\text {fin }}+\ln f_{0}^{1, \text { fin }}
$$

Since the sum of the natural logarithms of the distribution functions before and after the collision is the same, this relation shows that $\ln f$ is an invariant of the collision and can be expressed as the sum of the kinetic invariants:

$$
\ln f_{0}=\alpha_{0}+\alpha_{1} \cdot \xi+\alpha_{2} \xi^{2},
$$


where, on the right hand side, the first, second and third terms correspond to the conservation of the number of particles/total mass, momentum and energy, respectively. This may be rewritten in the following form:

$$
\ln f_{0}=\ln \beta_{0}-\beta_{1}\left(\boldsymbol{\xi}-\beta_{2}\right)^{2}
$$

for

$$
\beta_{0}=\exp \left(\alpha_{0}-\frac{\alpha_{1}^{2}}{4 \alpha_{2}}\right), \beta_{1}=-\alpha_{2}, \beta_{2}=-\frac{\alpha_{1}}{2 \alpha_{2}},
$$

or

$$
\alpha_{0}=\ln \beta_{0}-\beta_{1} \beta_{2}^{2}, \alpha_{1}=2 \beta_{1} \beta_{2}, \alpha_{2}=-\beta_{1}
$$

such that the distribution function has the form of a Gaussian function as follows [17]:

$$
f_{0}=\beta_{0} \exp \left[-\beta_{1}\left(\boldsymbol{\xi}-\boldsymbol{\beta}_{2}\right)^{2}\right] .
$$

The macroscopic observables, such as density, momentum and energy density $\epsilon$, are defined by the moments of the distribution function with respect to the particle velocity, i.e., by the integrations as follows:

$$
\begin{aligned}
\rho & =m \int \phi_{0}(\boldsymbol{\xi}) f_{0}(\mathbf{x}, \boldsymbol{\xi}, t) \mathrm{d}^{3} \xi \\
\rho \mathbf{u} & =m \int \phi_{1}(\boldsymbol{\xi}) f_{0}(\mathbf{x}, \boldsymbol{\xi}, t) \mathrm{d}^{3} \xi \\
\epsilon & =m \int \phi_{2}(\boldsymbol{\xi}) f_{0}(\mathbf{x}, \boldsymbol{\xi}, t) \mathrm{d}^{3} \xi
\end{aligned}
$$

where

$$
\phi_{j}(\xi)=\left[\phi_{0}, \phi_{1}, \phi_{2}\right]=\left[1, \xi, \xi^{2} / 2\right]
$$

are the kinetic invariants of the $i$ th moment, $\rho$ is the mass density, $m$ is the particle mass, $\mathbf{u}$ is the velocity field and $\epsilon$ is the energy density, as follows:

$$
\epsilon=\frac{3}{2} \frac{\rho}{m} k_{\mathrm{B}} T+\frac{1}{2} \rho u^{2} .
$$

In order to fulfill the conditions (13)-(15), Equation (12) has to take the form of a Maxwellian distribution as follows:

$$
f_{0}=\frac{\rho m^{1 / 2}}{\left(2 \pi k_{\mathrm{B}} T\right)^{3 / 2}} \exp \left\{-\frac{m}{2 k_{\mathrm{B}} T}(\boldsymbol{\xi}-\mathbf{u})^{2}\right\},
$$

which may be checked by plugging into the equation; see, for example, [14].

\section{The Complex-Valued Distribution Function}

As shown in the following, our main result is the direct incorporation of the electromagnetic interaction in the distribution function.

\subsection{Motivation}

In order to motivate this, we consider the simple case of a single particle with charge $q$ and mass $m$ moving in a homogeneous magnetic field with strength $B$, oriented, without loss of generality, along the $z$ axis, i.e., $\mathbf{B}=B \mathbf{e}_{z}$. As is well known from classical electrodynamics [18], the Lorentz Force $\mathbf{F}_{\mathrm{L}}$ is given by the following:

$$
\mathbf{F}_{\mathrm{L}}=q(\mathbf{E}+\mathbf{v} \times \mathbf{B})
$$

where $\mathbf{v}$ is the particle velocity, $\mathbf{E}$ is the electric and $\mathbf{B}$ is the magnetic field.

Here, it is important to note that the electric field behaves like a polar vector such that its contribution to the particle velocity can be described as the contribution to the drift velocity of an ensemble of charged particles. The magnetic field, on the other hand, is 
more complicated due to the axial behavior of the corresponding interaction and may be considered to be a contribution to the statistical velocity, as the particles only change their direction but preserve the value of velocity and energy. Therefore, from here on, we only discuss the contribution of the magnetic field.

In order to illustrate the effect of the magnetic field interaction, Equation (19) may be transformed into a system of differential equations as follows:

$$
m \frac{\partial v_{x}}{\partial t}=q v_{y} B, m \frac{\partial v_{y}}{\partial t}=-q v_{x} B, \frac{\partial v_{z}}{\partial t}=0
$$

This describes a circular motion parallel to the $x$-y-plane with the angular frequency $\omega=q B / m$. Note that $\omega \propto B$.

Following [19], we now introduce the complex notation by multiplying the second equation of (20) by $i$ and adding it to the first equation of (20), which, after minor rearrangements, gives the following:

$$
\frac{\partial}{\partial t}\left(v_{x}+i v_{y}\right)=-i \omega\left(v_{x}+i v_{y}\right)
$$

and may be solved, giving the following:

$$
v_{\mathrm{c}}(t)=v_{0} e^{-i \omega t}
$$

where $v_{c}=v_{x}+i v_{y}, v_{0}$ is the initial (real) velocity (here, without loss of generality, we omit a possible complex phase shift).

In order to assess the contribution of the magnetic field, we now consider the velocity change, compared to the unmagnetized case (i.e., $v_{c}=v_{0}=$ const) within an infinitesimal time interval $\mathrm{d} t$ (or, in a more general sense, the deflection, due to the magnetic field, compared to linear propagation over the time interval $\mathrm{d} t$ ):

$$
v_{\mathcal{c}}(\mathrm{d} t)=v_{0} e^{-i \omega \mathrm{d} t} \simeq v_{0}(1-i \omega \mathrm{d} t)=v_{0}-i \frac{q v_{0} \mathrm{~d} t}{m} B
$$

As one can see, the change in the movement from linear to circular due to the magnetic field indeed may be achieved by shifting the velocity along the imaginary axis, whereby the shift is proportional to the magnetic field.

\subsection{The Complex-Valued Maxwellian Distribution Function}

From the considerations above, which consider a single particle and a constant magnetic field, we now, for the general case, conclude that the quantity $\xi$ may be complex in order to introduce magnetic terms to the distribution function, i.e., the following:

$$
\xi=\Re \xi+i \Im \xi
$$

Substituting this into Equation (8) gives the relation for the complex-valued Maxwellian distribution function in $f_{0}^{\prime}$ as follows:

$$
\begin{aligned}
\ln f_{0}^{\prime} & =\alpha_{0}^{\prime}+\alpha_{1}^{\prime} \cdot \xi+\alpha_{2}^{\prime} \xi^{2}=\alpha_{0}^{\prime}+\alpha_{1}^{\prime} \cdot(\Re \xi+i \Im \xi)+\alpha_{2}^{\prime} \xi^{2} \\
& =\alpha_{0}^{\prime}+\alpha_{1}^{\prime} \cdot \Re \xi+i \alpha_{1}^{\prime} \cdot \Im \xi+\alpha_{2}^{\prime}(\Re \xi+i \Im \xi)^{2},
\end{aligned}
$$

Therefore, we now have four terms corresponding to four invariants:

- $\quad \alpha_{0}^{\prime}$ represents the number of particles/total mass;

- $\quad \alpha_{1}^{\prime} \cdot \Re \xi$ stands for the momentum density;

- $\quad i \alpha_{1}^{\prime} \cdot \Im \xi$ is connected to the magnetic field;

- $\quad \alpha_{2}^{\prime}(\Re \xi+i \Im \xi)^{2}$ is related to the (total) energy density, now also including the magnetic contributions. 
Note that for the magnetic field itself and the energy density term, the invariants are complex, which is due to the fact that in both cases, the (axial) vector behavior plays a role for their time evolution (s. below). This confirms the validity of our approach.

On the other hand, the transformation (24) may be also carried out directly for expression (12), giving the following:

$$
f_{0}^{\prime}=\beta_{0}^{\prime} \exp \left[-\beta_{1}^{\prime}\left(\xi-\beta_{2}^{\prime}\right)^{2}\right]=\beta_{0}^{\prime} \exp \left[-\beta_{1}^{\prime}\left(\Re \xi+i \Im \xi-\beta_{2}^{\prime}\right)^{2}\right] .
$$

\subsection{Moments of the Complex-Valued Boltzmann Distribution Function}

In order to have the most general form of the integration invariants for the moments of the Boltzmann equation, i.e., taking into account possible complex contributions that are not present in the case of real variables, we generalize them from the form given in (16) as follows:

$$
\phi_{j}(\xi)=\left[1, \xi, \xi^{2} / 2\right]
$$

to

$$
\phi_{j}^{\prime}(\xi)=\left[\phi_{0}^{\prime}, \phi_{1}^{\prime}, \phi_{2}^{\prime}\right]=\left[1, \xi+i \gamma_{1}^{\prime} \Im \xi, \frac{1}{2}\left(\xi^{2}+i \gamma_{2}^{\prime} \Re \xi \cdot \Im \xi\right)\right]
$$

From the interpretation of the new invariants above, we will now derive the values for the different $\beta_{i}^{\prime}$ introduced in (26) from the moments of the distribution function $f_{0}$. It should be noted here that the introduction of $\phi_{j}^{\prime}$ as (28) is the most general form of the extension of (16) to the complex plane. In contrast to previous works [7-12], in the following, we will derive the most general conditions on the parameters $\gamma_{1}^{\prime}$ and $\gamma_{2}^{\prime}$, while also providing a convenient choice for them for illustration purposes.

The set of conditions for the 0th moment is given by the following:

$$
\begin{aligned}
& m \Re \int \phi_{0}^{\prime} f_{0}^{\prime} \mathrm{d}^{3} \xi=m \Re \int f_{0}^{\prime} \mathrm{d}^{3} \xi=\rho, \\
& m \Im \int \phi_{0}^{\prime} f_{0}^{\prime} \mathrm{d}^{3} \xi=m \Im \int f_{0}^{\prime} \mathrm{d}^{3} \xi=0,
\end{aligned}
$$

of which the latter one arises due to the fact that the integral of a distribution function over $\xi$ is interpreted as the cumulative probability and hence, has to be real, while the former condition ensures the normalization of $f_{0}$ such that the total probability is equal to 1 .

Therefore, using the standard rules of integration of complex-valued Gaussian functions (cf. [20]), one obtains the following:

$$
m \int \phi_{0}^{\prime} f_{0}^{\prime} \mathrm{d}^{3} \xi=m \int 1 \cdot f_{0}^{\prime} \mathrm{d}^{3} \xi=\int m \beta_{0}^{\prime} \exp \left[-\beta_{1}^{\prime}\left(\boldsymbol{\xi}-\boldsymbol{\beta}_{2}^{\prime}\right)^{2}\right] \mathrm{d}^{3} \xi=m \beta_{0}^{\prime} \sqrt{\frac{\pi^{3}}{\beta_{1}^{\prime}}}
$$

from which, using (29), we can conclude the following:

$$
m \beta_{0}^{\prime} \sqrt{{\frac{\pi}{\beta_{1}^{\prime}}}^{3}}=\rho,
$$

and, using (30), we have the following:

$$
\beta_{0}^{\prime}, \beta_{1}^{\prime} \in \mathbb{R} .
$$

The requirements on the first moment are given by the following:

$$
\begin{aligned}
& m \Re \int \phi_{1}^{\prime} f_{0}^{\prime} \mathrm{d}^{3} \xi=\Re \int m\left(\xi+i \gamma_{1}^{\prime} \Im \xi\right) f_{0} \mathrm{~d}^{3} \xi=\rho \mathbf{u}, \\
& m \Im \int \phi_{1}^{\prime} f_{0}^{\prime} \mathrm{d}^{3} \xi=\Im \int m\left(\xi+i \gamma_{1}^{\prime} \Im \xi\right) f_{0} \mathrm{~d}^{3} \xi=\kappa^{\prime} \rho \mathbf{v}_{\mathrm{A}},
\end{aligned}
$$


where $\mathbf{v}_{\mathrm{A}}$ is the Alfvén velocity given by the following:

$$
\mathbf{v}_{\mathrm{A}}=\frac{\mathbf{B}}{\sqrt{\mu_{0} \rho}}
$$

for the magnetic field strength $\mathbf{B}$ and the vacuum permeability $\mu_{0} ; \kappa^{\prime}$ is a factor connected to the general ratio of magnetic to kinetic terms. The introduction of the magnetic field using the Alfvén velocity is a natural choice, it being a key quantity in MHD, characterizing a so-called Alfvén wave, which is a fundamental phenomenon in magnetized plasmas [21]. Note, however, that it is also possible to carry out this introduction of the magnetic field using another quantity with the dimension of a velocity, as long as it is proportional to $\mathbf{B}$, which was motivated in Section 3.1.

For the first moment (i.e., for $\phi_{1}^{\prime}$ ), again, using the rules for Gaussian integration, we obtain the following:

$$
\begin{aligned}
m \int \phi_{1}^{\prime} f_{0}^{\prime} \mathrm{d}^{3} \xi & =m \int\left(\boldsymbol{\xi}+i \gamma_{1}^{\prime} \Im \xi\right) f_{0} \mathrm{~d}^{3} \xi=m \int\left(\boldsymbol{\xi}+i \gamma_{1}^{\prime} \Im \xi\right) \beta_{0} \exp \left[-\beta_{1}^{\prime}\left(\xi-\boldsymbol{\beta}_{2}^{\prime}\right)^{2}\right] \mathrm{d}^{3} \xi \\
& =m \beta_{0}^{\prime} \sqrt{\frac{\pi^{3}}{\beta_{1}^{\prime}}}\left(\boldsymbol{\beta}_{2}^{\prime}+i \gamma_{1}^{\prime} \Im \boldsymbol{\beta}_{2}^{\prime}\right),
\end{aligned}
$$

which, using (32), becomes

$$
m \int\left(\boldsymbol{\xi}+i \gamma_{1}^{\prime} \Im \xi\right) f_{0} \mathrm{~d}^{3} \xi=\rho\left(\boldsymbol{\beta}_{2}^{\prime}+i \gamma_{1}^{\prime} \Im \boldsymbol{\beta}_{2}^{\prime}\right),
$$

and, therefore, from (34) and (35), we obtain the following:

$$
\boldsymbol{\beta}_{2}^{\prime}=\mathbf{u}+i \frac{\kappa^{\prime}}{1+\gamma_{1}^{\prime}} \mathbf{v}_{\mathrm{A}}=\mathbf{u}+i \frac{\kappa^{\prime}}{1+\gamma_{1}^{\prime}} \frac{\mathbf{B}}{\sqrt{\mu_{0} \rho}},
$$

implying the condition $\beta_{2}^{\prime} \in \mathbb{C}^{3}$. This is a very important conclusion since, as may be seen from this together with (33), it means that $\beta_{2}$ is the only complex factor in the distribution function and, therefore, is solely responsible for the shift of the result along the imaginary axis. Furthermore, since the choice of $\kappa^{\prime}$ does not influence the validity of the distribution function (as $\beta_{2}$ does not appear in condition (32)), it may be chosen arbitrarily for each integration; here, this means that each moment has its own $\kappa^{\prime}$ value, which we hereinafter denote as $\kappa_{j}^{\prime}$, where $j$ is the number of the moment.

In order to have the most convenient form for the expression of the magnetic field, we choose $\gamma_{1}^{\prime}=0$, and, furthermore, in order to obtain the magnetic field itself, the following:

$$
\kappa_{1}^{\prime}=\kappa_{1}^{\prime \prime} \sqrt{\frac{\rho}{\mu_{0}}},
$$

where $\kappa_{1}^{\prime \prime}$ is an arbitrary constant (i.e., independent of $\mathbf{x}$ and $t$ ).

Finally, for the third moment, we have the following requirement:

$$
m \Re \int \phi_{2}^{\prime} f_{0}^{\prime} \mathrm{d}^{3} \xi=m \Re \int \frac{1}{2}\left(\xi^{2}+i \gamma_{2}^{\prime} \Re \xi \cdot \Im \xi\right) f_{0} \mathrm{~d}^{3} \xi=\epsilon,
$$

for which $\epsilon$ is the total energy density given by the following:

$$
\epsilon=\frac{3}{2} p+\frac{1}{2} \rho u^{2}+\frac{\mathbf{B}^{2}}{2 \mu_{0}},
$$


where the three terms correspond to the internal, kinetic and magnetic energy, respectively, with the following being the hydrodynamic pressure:

$$
p=\frac{\rho}{m} k_{\mathrm{B}} T
$$

It is more convenient, however, to express $\epsilon$ in terms of the total pressure $P$ given by the following:

$$
P=p+\frac{\mathbf{B}^{2}}{2 \mu_{0}},
$$

such that (42) may be expressed as follows:

$$
\epsilon=\frac{3}{2} P+\frac{1}{2} \rho u^{2}-\frac{\mathbf{B}^{2}}{4 \mu_{0}} .
$$

Then, the second moment, again using Gaussian integration, yields the following:

$$
\begin{aligned}
m \Re \int \frac{1}{2}\left(\xi^{2}+i \gamma_{2}^{\prime} \Re \xi \cdot \Im \xi\right) f_{0} \mathrm{~d}^{3} \xi & =m \Re \int \frac{1}{2}\left(\xi^{2}+i \gamma_{2}^{\prime} \Re \xi \cdot \Im \xi\right) \beta_{0}^{\prime} \exp \left[-\beta_{1}^{\prime}\left(\xi-\beta_{2}^{\prime}\right)^{2}\right] \mathrm{d}^{3} \xi \\
& =\frac{1}{2} m \beta_{0}^{\prime} \sqrt{\frac{\pi^{3}}{\beta_{1}^{\prime}}}\left[\left(\frac{3}{2 \beta_{1}^{\prime}}+\boldsymbol{\beta}_{2}^{\prime 2}\right)\right],
\end{aligned}
$$

which, using (32) and (39), gives the following:

$$
m \Re \int \frac{1}{2}\left(\xi^{2}+i \gamma_{2}^{\prime} \Re \xi \cdot \Im \xi\right) f_{0} \mathrm{~d}^{3} \xi=\frac{\rho}{2}\left[\left(\frac{3}{2 \beta_{1}^{\prime}}+\mathbf{u}^{2}-\kappa_{2}^{\prime 2} \frac{\mathbf{B}^{2}}{\mu_{0} \rho}\right)\right],
$$

such that from (41), we obtain the following:

$$
\frac{3}{2} P-\frac{\mathbf{B}^{2}}{4 \mu_{0}}=\frac{3 \rho}{4 \beta_{1}^{\prime}}-\kappa_{2}^{\prime 2} \frac{\mathbf{B}^{2}}{2 \mu_{0}},
$$

which may be transformed into the following:

$$
\beta_{1}^{\prime}=\left[2 \frac{P}{\rho}+\frac{1}{3}\left(2 \kappa_{2}^{\prime 2}-1\right) \frac{\mathbf{B}^{2}}{\mu_{0} \rho}\right]^{-1} .
$$

In order for the equation to reproduce the correct energy density, we choose $\kappa_{2}^{\prime}=1 / \sqrt{2}$ such that we obtain the following simple relation:

$$
\beta_{1}^{\prime}=\frac{\rho}{2 P} .
$$

and therefore, from (32), we obtain the following:

$$
\beta_{0}^{\prime}=\frac{\rho}{m} \sqrt{\frac{\rho}{2 \pi P}}^{3},
$$

such that the equilibrium function $f_{0}^{\prime}$ from (26) becomes the following:

$$
f_{0}^{\prime}=\frac{\rho m^{1 / 2}}{\left(2 \pi k_{\mathrm{B}} T^{\prime}\right)^{3 / 2}} \exp \left\{-\frac{m}{2 k_{\mathrm{B}} T^{\prime}}\left[\boldsymbol{\xi}-\left(\mathbf{u}+i \kappa^{\prime} \mathbf{v}_{\mathrm{A}}\right)\right]^{2}\right\},
$$

where $T^{\prime}$ is the temperature including the magnetic term, i.e., the following:

$$
T^{\prime}=\frac{m P}{k_{\mathrm{B}} \rho}=\frac{m\left(p+\frac{\mathbf{B}^{2}}{2 \mu_{0}}\right)}{k_{\mathrm{B}} \rho} .
$$




\subsection{Moments of the Boltzmann Equation for the Complex-Valued Distribution Function}

Before calculating the moments of the Boltzmann Equation, we summarize the findings of the previous section. The form of the new complex distribution function is given by the following:

$$
f_{0}^{\prime}=\frac{\rho m^{1 / 2}}{\left(2 \pi k_{\mathrm{B}} T^{\prime}\right)^{3 / 2}} \exp \left\{-\frac{m}{2 k_{\mathrm{B}} T^{\prime}}\left[\xi-\left(\mathbf{u}+i \kappa^{\prime} \mathbf{v}_{\mathrm{A}}\right)\right]^{2}\right\},
$$

and the corresponding quantities may be calculated from its moments as follows:

$$
\begin{aligned}
\rho & =\Re \int m f_{0}^{\prime} \mathrm{d}^{3} \xi, \\
0 & =\Im \int m f_{0}^{\prime} \mathrm{d}^{3} \xi, \\
\rho \mathbf{u} & =\Re \int m \xi f_{0}^{\prime} \mathrm{d}^{3} \xi, \\
\mathbf{B} & =\frac{1}{\kappa_{1}^{\prime}} \Im \int m \xi f_{0} \mathrm{~d}^{3} \xi, \\
\epsilon & =\Re \int \frac{1}{2} m\left(\xi^{2}+i \gamma_{2}^{\prime} \Re \xi \cdot \Im \xi\right) f_{0} \mathrm{~d}^{3} \xi
\end{aligned}
$$

In order to obtain the time evolution equations, we now calculate the same moments for the Boltzmann equation. The zeroth moment (i.e., for $\phi_{0}^{\prime}$ ) of the Boltzmann Equation (1) for $C(f)=0$ gives the following relation:

$$
\int m \frac{\partial f_{0}^{\prime}(\mathbf{x}, \boldsymbol{\xi}, t)}{\partial t} \mathrm{~d}^{3} \xi+\int m \xi \cdot \nabla f_{0}^{\prime}(\mathbf{x}, \boldsymbol{\xi}, t) \mathrm{d}^{3} \xi=0,
$$

which, after carrying out the integral, gives the following:

$$
\frac{\partial \rho}{\partial t}+\nabla \cdot\left(\rho \mathbf{u}+i \kappa_{0}^{\prime} \sqrt{\frac{\rho}{\mu_{0}}} \mathbf{B}\right)=0,
$$

where the concept of $\kappa^{\prime}$ is introduced in Equation (35), while the $\kappa_{j}^{\prime}$ is discussed after Equation (39). Considering the real and imaginary parts, as was done in (29) and (30), and furthermore setting $\kappa_{0}^{\prime}=\kappa_{0}^{\prime \prime} \sqrt{\mu_{0} / \rho}$, where $\kappa_{0}^{\prime \prime}$ is independent of $\mathbf{x}$ and $t$, one obtains the two following relations:

$$
\begin{aligned}
\frac{\partial \rho}{\partial t}+\nabla \cdot(\rho \mathbf{u}) & =0, \\
\nabla \cdot \mathbf{B} & =0,
\end{aligned}
$$

which correspond to the (mass) continuity equation and the divergence freedom of the magnetic field, respectively.

The first moment of the Boltzmann equation (i.e., for $\phi_{1}^{\prime}$ ) is calculated according to the following:

$$
\int m\left(\xi+i \gamma_{1}^{\prime} \Im \xi\right) \frac{\partial f_{0}(\mathbf{x}, \boldsymbol{\xi}, t)}{\partial t} \mathrm{~d}^{3} \xi+\int m\left(\xi+i \gamma_{1}^{\prime} \Im \xi\right) \xi \cdot \nabla f_{0}(\mathbf{x}, \xi, t) \mathrm{d}^{3} \xi=0,
$$

which, for the different components $i$, results in the following:

$$
\frac{\partial}{\partial t}\left[\rho u_{i}+i \kappa_{1}^{\prime} \sqrt{\frac{\rho}{\mu_{0}}} \mathbf{B}\right]+\frac{\partial}{\partial x_{j}}\left[\left(P \delta_{i j}+\rho u_{i} u_{j}-\frac{B_{i} B_{j}}{\mu_{0}}\right)+i \kappa_{1}^{\prime}\left(u_{j} B_{i}-u_{i} B_{j}\right)\right]=0 .
$$


Taking the real and imaginary parts of this equation, together with (40), then gives the following equations:

$$
\begin{aligned}
\frac{\partial\left(\rho u_{i}\right)}{\partial t}+\frac{\partial}{\partial x_{j}}\left[\left(P \delta_{i j}+\rho u_{i} u_{j}-\frac{B_{i} B_{j}}{\mu_{0}}\right)\right] & =0, \\
\frac{\partial B_{i}}{\partial t}+\frac{\partial}{\partial x_{j}}\left[\left(u_{j} B_{i}-u_{i} B_{j}\right)\right] & =0
\end{aligned}
$$

which correspond to the momentum and the induction equation, respectively. Note that the Einstein summation is used in Equations (65)-(67).

Finally, the second moment of the Boltzmann equation (i.e., for $\phi_{2}^{\prime}$ ) is calculated, according to (59) as follows:

$\Re \int \frac{1}{2} m\left(\xi^{2}+i \gamma_{2}^{\prime} \Re \xi \cdot \Im \xi\right) \frac{\partial f_{0}^{\prime}(\mathbf{x}, \xi, t)}{\partial t} \mathrm{~d}^{3} \xi+\Re \int \frac{1}{2} m\left(\xi^{2}+i \gamma_{2}^{\prime} \Re \xi \cdot \Im \xi\right) \xi \cdot \nabla f_{0}^{\prime}(\mathbf{x}, \xi, t) \mathrm{d}^{3} \xi=0$,

which, using $\kappa_{2}^{\prime}=1 / \sqrt{2}$ from above and setting $\gamma_{2}^{\prime}=2$ (thus fixing the last unknown parameter) results in the following:

$$
\frac{\partial}{\partial t}\left[\frac{3}{2} P+\frac{1}{2} \rho \mathbf{u}^{2}-\frac{\mathbf{B}^{2}}{4 \mu_{0}}\right]+\frac{\partial}{\partial x_{j}}\left[\left(\frac{5}{2} P+\frac{1}{2} \rho \mathbf{u}^{2}-\frac{\mathbf{B}^{2}}{4 \mu_{0}}\right) u_{j}-\mathbf{u} \cdot \mathbf{B} \frac{B_{j}}{\mu_{0}}\right]=0,
$$

which corresponds to the energy conservation equation [22].

\section{Conclusions and Outlook}

In this work, we derived and verified a novel complex-valued Maxwellian distribution function $f_{0}^{\prime}$, for which the imaginary part represents the magnetic fields directly as an intrinsic part of the distribution function. This is the central result of our work, as until now, in order to derive the equations of MHD, it was necessary to introduce the magnetic field as an external interaction. This is insofar unsatisfactory, as both the magnetic fields and the kinetic quantities are integral components of MHD and, therefore, there is no conceptual reason to consider the former as external and the latter as internal contributions. Here, we have presented an elegant way to combine both into a unified formalism. Since our method may be considered a generalization of the hydrodynamic case, in the future, kinetic consistent schemes, for example, may be used to derive all possible terms of non-ideal MHD, similar to what was done for hydrodynamics. Again, this enables us to derive all possible viscous terms directly from the Boltzmann equation instead of introducing them as external terms, i.e., in the most natural way, thus validating and extending the current approach.

It is important to note that the introduction of complex quantities is necessary to take into account the axial-vector behavior of the magnetic field. In particular, in this work, we presented the full physics derivation from first principles and obtained the most general form of the new distribution function, which does not require any further assumptions, given by the following:

$$
f_{0}^{\prime}=\frac{\rho m^{1 / 2}}{\left(2 \pi k_{\mathrm{B}} T^{\prime}\right)^{3 / 2}} \exp \left\{-\frac{m}{2 k_{\mathrm{B}} T^{\prime}}\left[\boldsymbol{\xi}-\left(\mathbf{u}+i \kappa^{\prime} \mathbf{v}_{\mathrm{A}}\right)\right]^{2}\right\},
$$

with the summation invariants to calculate its corresponding moments being the following:

$$
\phi_{j}(\xi)^{\prime}=\left[\phi_{0}^{\prime}(\xi), \phi_{1}^{\prime}(\xi), \phi_{2}^{\prime}(\xi)\right]=\left[1, \xi, \frac{1}{2}\left(\xi^{2}+2 i \Re \xi \cdot \Im \xi\right)\right],
$$

and the corresponding $\kappa_{j}^{\prime}$-values being the following:

$$
\kappa_{j}^{\prime}=\left[\kappa_{0}^{\prime}, \kappa_{1}^{\prime}, \kappa_{2}^{\prime}\right]=\left[\kappa_{0}^{\prime \prime} \sqrt{\mu_{0} / \rho}, \kappa_{1}^{\prime \prime} \sqrt{\mu_{0} / \rho}, 1 / \sqrt{2}\right],
$$


where $\kappa_{0}^{\prime \prime}$ and $\kappa_{1}^{\prime \prime}$ are arbitrary constants such that the corresponding dimensions are given by $\left[\kappa_{0}^{\prime \prime} \sqrt{\mu_{0} / \rho}\right]=\left[\kappa_{1}^{\prime \prime} \sqrt{\mu_{0} / \rho}\right]=1$.

It was furthermore shown that calculating the corresponding moments of the Boltzmann equation using $f_{0}^{\prime}$ gives the correct equations of ideal MHD, thereby confirming the correctness of our approach. This is, therefore, the most natural way to introduce magnetic fields since by doing so all relevant quantities of MHD are introduced via the distribution function itself and not via an external interaction. It should be noted here that for the way we have constructed the complex-valued distribution function and the corresponding mechanism, the classical Boltzmann equation remains valid, the only difference being that the velocity $\xi$ is assumed to be complex instead of real. The proposed method of the complex-valued distribution function may be applied to solve MHD problems by applying KCS and LBS on high-performance computational systems [23].

In the future, it would be interesting to investigate the possibility of extending our formalism to the relativistic case. This, in principle, is straightforward; however, it is conceivable for the parameters $\gamma_{j}^{\prime}$ and $\kappa_{j}^{\prime}$ to have different conditions defining them for which the relations found in this work will be the limiting case for non-relativistic velocities. This, however, requires a full retracing of every step (and its validity) presented here (which might be challenging, due to the more complicated mathematics involved), starting from the relativistic form of the distribution function and the corresponding Boltzmann equation, for example, in the form presented in [24]. Again, while previous approaches of considering magnetic fields within the kinetic framework of the distribution function do exist (see, for example, $[25,26]$ ), it would be desirable to obtain the equations of relativistic MHD from first principles, with the magnetic field being an intrinsic property of the distribution function.

Another interesting aspect to be studied is the relevance of quantum-mechanical effects. Again, this is possible by starting off with the basic form of the formalism, i.e., with the Uehling-Uhlenbeck equation for which the collision integral is modified in order to accommodate, in particular, the effect of the spin on the collision process. By subsequently introducing the complex-valued distribution function and calculating the corresponding moments, in principle, the modifications of MHD by quantum physics may be calculated.

Funding: This work was supported by the Ministry of Science and Higher Education of the Russian Federation (agreement no. 075-02-2021-1748).

Institutional Review Board Statement: Not applicable.

Informed Consent Statement: Not applicable.

Data Availability Statement: Not applicable.

Acknowledgments: The author is grateful to Boris Chetverushkin, Academician of the Russian Academy of Sciences, for valuable comments during the completion of this work.

Conflicts of Interest: The author declares no conflict of interest.

\section{Abbreviations}

The following abbreviations are used in this manuscript:

KCS Kinetic Consistent Schemes

LBS Lattice Boltzmann Schemes

MHD Magnetohydrodynamics

\section{References}

1. Boltzmann, L. Lectures on Gas Theory; University of California Press: Oakland, CA, USA, 1964.

2. Vlasov, A.A. The Vibrational Properties of an Electron Gas. Zh. Eksp. Teor. Fiz. 1938, 8, 291. [CrossRef]

3. Croisille, J.P.; Khanfir, R.; Chanteur, G. Numerical Simulation of the MHD Equations by a Kinetic-Type Method. J. Sci. Comp. 1995, 10, 81-92. [CrossRef] 
4. Huba, J.D.; Lyon, J.G. A New 3D MHD Algorithm: The Distribution Function Method. J. Plasma Phys. 1999, 61, $391-405$. [CrossRef]

5. Dellar, P.J. Lattice Kinetic Schemes for Magnetohydrodynamics. J. Comp. Phys. 2002, 179, 95-126. [CrossRef]

6. Bouchut, F. Construction of BGK Models with a Family of Kinetic Entropies for a Given System of Conservation Laws. J. Stat. Phys. 1999, 95, 113-170. [CrossRef]

7. Chetverushkin, B.N.; D'Ascenzo, N.; Saveliev, V.I. Kinetically Consistent Magnetogasdynamics Equations and their Use in Supercomputer Computations. Dokl. Math. 2014, 90, 495-498. [CrossRef]

8. Chetverushkin, B.N.; D'Ascenzo, N.; Saveliev, A.V.; Saveliev, V.I. Novel Kinetically Consistent Algorithm for Magneto Gas Dynamics. Appl. Math. Lett. 2017, 72, 75-81. [CrossRef]

9. Chetverushkin, B.N.; Saveliev, A.V.; Saveliev, V.I. A Quasi-Gasdynamic Model for the Description of Magnetogasdynamic Phenomena. Comp. Math. Math. Phys. 2018, 58, 1384-1394. [CrossRef]

10. Chetverushkin, B.; D’Ascenzo, N.; Ishanov, S.; Saveliev, V. Hyperbolic Type Explicit Kinetic Scheme of Magneto Gas Dynamics for High Performance Computing Systems. Rus. J. Num. Anal. Math. Mod. 2015, 30, 27-36. [CrossRef]

11. Chetverushkin, B.N.; Saveliev, A.V.; Saveliev, V.I. Compact Quasi-Gasdynamic System for High-Performance Computations. Comp. Math. Math. Phys. 2019, 59, 493-500. [CrossRef]

12. Chetverushkin, B.N.; Saveliev, A.V.; Saveliev, V.I. Kinetic Algorithms for Modeling Conductive Fluids Flow on High-Performance Computing Systems. Dokl. Math. 2019, 100, 577-581. [CrossRef]

13. Succi, S. The Lattice Boltzmann Equation for Fluid Mechanics and Beyond; Oxford University Press: Oxford, UK, 2001.

14. Chetverushkin, B.N. Kinetic Schemes and Quasi-Gas Dynamic System of Equations; CIMNE: Barcelona, Spain, 2008.

15. Boltzmann, L. Weitere Studien über das Wärmegleichgewicht unter Gasmolekülen. Kinetische Theorie II 1872, 66, $275-370$.

16. Gombosi, T.I. Gaskinetic Theory; Cambridge University Press: Cambridge, UK, 1994.

17. Landau, L.D.; Lifshitz, E.M. Physical Kinetics; Course of Theoretical Physics; Pergamon Press: Oxford, UK, 1971; Volume 10.

18. Jackson, J.D. Classical Electrodynamics; John Wiley \& Sons: Hoboken, NJ, USA, 1998.

19. Landau, L.D.; Lifshitz, E.M. The Classical Theory of Fields; Course of Theoretical Physics; Pergamon Press: Oxford, UK, 2011; Volume 2.

20. Bronstein, I.N.; Semendyayev, K.A.; Musiol, G.; Muehlig, H. Handbook of Mathematics; Springer: Berlin/Heidelberg, Germany, 2007.

21. Alfvén, H. Existence of Electromagnetic-Hydrodynamic Waves. Nature 1942, 150, 405-406. [CrossRef]

22. Webb, G. Magnetohydrodynamics and Fluid Dynamics: Action Principles and Conservation Laws; Springer: Berlin/Heidelberg, Germany, 2018.

23. Ahern, S. Scientific Application Requirements for Leadership Computing at the Exascale; Technical Report TM-2007/238; ORNL: Oak Ridge, TN, USA, 2007.

24. Cercignani, C.; Kremer, G.M. The Relativistic Boltzmann Equation: Theory and Applications; Springer: Berlin/Heidelberg, Germany, 2002.

25. Goto, K. Relativistic Magnetohydrodynamics. Prog. Theor. Phys. 1958, 20, 1-14. [CrossRef]

26. Tam, K.; Kiang, D. The Relativistic Boltzmann Equation and the Equations of Magnetohydrodynamics with Radiative Reaction. Prog. Theor. Phys. 1979, 62, 1245-1252. [CrossRef] 